\title{
Almost Contact $B$-metric Structure on 5-Dimensional Nilpotent Lie Algebras
}

\author{
Şenay Bulut* and Sevgi Enveş Ermiş
}

(Communicated by Cihan Özgür)

\begin{abstract}
The classification of almost contact $B$-metric manifolds is considered. It is shown that $\mathcal{D}$-homothetic deformation of these manifolds in the class $\mathcal{F}_{i}(i=0,1,4,5)$ remains in the same the class $\mathcal{F}_{i}$. We study almost contact $B$-metric structure on 5 -dimensional nilpotent Lie algebras. The class of the left invariant almost contact $B$-metric structures on corresponding Lie groups is investigated. Finally, we determine the class of 5 -dimensional nilpotent Lie algebras with almost contact $B$-metric structure.
\end{abstract}

Keywords: 5-dimensional nilpotent Lie algebra; Almost contact B-metric manifold; Fundamental tensor.

AMS Subject Classification (2020): Primary: 53C05 ; Secondary: 53C15; 53C25.

\section{Introduction}

The classifications of almost contact $B$-metric manifolds and almost contact metric manifolds were obtained in $[2,4,5,7]$. The eleven basic classes of almost contact $B$-metric manifolds were obtained by using the structure tensor of type $(0,3)$ [5]. In [1] the concept of $\mathcal{D}$-homothetic deformation of an almost contact $B$-metric manifolds is introduced and some properties of these are investigated. Left invariant almost contact metric structure on every connected odd dimensional Lie group give rise to almost contact metric structure on corresponding Lie algebras [8]. The classification of 5-dimensional nilpotent Lie algebras with almost contact metric structure is given in [3].

Our aim in this paper is to determine the class of 5 -dimensional nilpotent Lie algebras with respect to the given almost contact $B$-metric structure. In Section 2 we recall some facts about the almost contact $B$-metric manifolds and it is shown that $\mathcal{D}$-homothetic deformation of these manifolds within a certain class remains within the same class. In Section 3 we give almost contact $B$-metric structure on 5 -dimensional nilpotent Lie algebras $\mathfrak{g}_{i}$ for $i=1, \ldots, 6$. We determine the class of 5 -dimensional nilpotent Lie algebras $\mathfrak{g}_{i}$ with this structure. Finally, it is proved that the vector field $\xi$ is a Killing vector field for each $\mathfrak{g}_{i}$.

\section{Almost Contact $B$-metric Manifolds}

Let $M$ be a smooth manifold of dimension $(2 n+1)$. If $M$ has an almost contact structure $(\varphi, \eta, \xi)$ consisting of an endomorphism $\varphi$ of the tangent bundle, a Reeb vector field $\xi$, its dual 1 -form $\eta$ such that the following relations are satisfied

$$
\varphi^{2}=-I d+\eta \otimes \xi, \quad \eta(\xi)=1,
$$

then, $(M, \varphi, \eta, \xi)$ is called almost contact manifold. In addition, if $(M, \varphi, \xi, \eta)$ admits a pseudo-Riemannian metric $g$ of signature $(n+1, n)$ such that

$$
g(\varphi x, \varphi y)=-g(x, y)+\eta(x) \eta(y)
$$


for all vector fields $x, y$, then, $(M, \varphi, \xi, \eta, g)$ is called almost contact $B$-metric manifold.

The horizontal distribution induced by the contact $1-$ form $\eta$ is denoted by $H=k e r \eta$. Taking the restrictions of $\varphi$ to $H$ and $g$ to $H,\left(H,\left.\varphi\right|_{H},\left.g\right|_{H}\right)$ can be considered as an almost complex manifold with Norden metric of dimension $2 n$.

The structure group of the almost contact $B$-metric manifolds is $O(n, \mathbb{C}) \times 1$, that is, $O(n, \mathbb{C}) \times 1$ consists of $(2 n+1) \times(2 n+1)$ matrices of the following type

$$
\left(\begin{array}{ccc}
A & B & 0_{n \times 1} \\
-B & A & 0_{n \times 1} \\
0_{1 \times n} & 0_{1 \times n} & 1
\end{array}\right), \quad A A^{t}-B B^{t}=I_{n}, \quad A B^{t}+B A^{t}=0_{n},
$$

where $A, B \in G L(n, \mathbb{R})$ and $I_{n}$ and $0_{n}$ are the unit matrix and zero matrix, respectively.

In [5] the almost contact $B$-metric manifolds are classified using the tensor $F$ of type $(0,3)$ defined by

$$
F(x, y, z)=g\left(\left(\nabla_{x} \varphi\right) y, z\right)
$$

where $\nabla$ is the Levi-Civita connection of $g$. Moreover, the following properties of $F$ are valid:

$$
\begin{aligned}
& F(x, y, z)=F(x, z, y)=F(x, \varphi y, \varphi z)+\eta(y) F(x, \xi, z)+\eta(z) F(x, y, \xi), \\
& \left(\nabla_{x} \eta\right) y=g\left(\nabla_{x} \xi, y\right)=F(x, \varphi y, \xi) .
\end{aligned}
$$

Eleven basis classes of these manifolds are denoted by $\mathcal{F}_{1}, \ldots, \mathcal{F}_{11}$. The class of $\mathcal{F}_{0}$ is defined by the condition $F=0$, i.e., $\nabla \varphi=\nabla \xi=\nabla \eta=\nabla g=0$.

The Lie 1 -forms associated with $F$ are defined by

$$
\theta(x)=g^{i j} F\left(e_{i}, e_{j}, x\right), \quad \theta^{*}(x)=g^{i j} F\left(e_{i}, \varphi e_{j}, x\right), \quad \omega(x)=F(\xi, \xi, x),
$$

where $g^{i j}$ 's are the entries of the inverse matrix of $g$ with respect to the basis $\left\{e_{i}, \xi\right\}$ of $T_{p} M$.

An almost contact $B$-metric structure $(\varphi, \eta, \xi)$ on a connected Lie group $G$ is said to be left invariant if $g$ is the left invariant and if the left multiplication map $L_{a}: G \rightarrow G$ has the following properties

$$
\varphi \circ d L_{a}=d L_{a} \circ \varphi \text { and } d L_{a}(\xi)=\xi
$$

for all $a \in G$.

Let $\mathfrak{g}$ be odd dimensional Lie algebra. An almost contact $B$-metric structure on $\mathfrak{g}$ consists of a one-form $\eta$, a tensor field $\varphi$, a vector field $\xi$ such that

$$
\varphi^{2}=-I d+\eta \otimes \xi, \quad \eta(\xi)=1, \quad g(\varphi x, \varphi y)=-g(x, y)+\eta(x) \eta(y),
$$

for all vector fields $x, y$ where $g$ is a $B$-metric.

\subsection{D-homothetic Deformation on Almost Contact B-metric Structures}

Let $(M, \varphi, \xi, \eta, g)$ be an almost contact $B$-metric manifold. The structure $(\widetilde{\varphi}, \widetilde{\xi}, \widetilde{\eta}, \widetilde{g})$ satisfying the relations

$$
\widetilde{\eta}=t \eta, \quad \widetilde{\xi}=\frac{1}{t} \xi, \quad \widetilde{\varphi}=\varphi, \quad \widetilde{g}=-t g+t(t+1) \eta \otimes \eta,
$$

with the positive constant $t$ is called a $\mathcal{D}$-homothetic deformation of $(M, \varphi, \xi, \eta, g)$.

The relation between the tensors $F$ and $\widetilde{F}$ can be found in [1]:

$$
\begin{aligned}
\widetilde{F}(x, y, z)=-t F(x, y, z)+\frac{t(t+1)}{2} & \{d \eta(\varphi y, z) \eta(x)-d \eta(y, \varphi z) \eta(x) \\
& -d \eta(x, \varphi y) \eta(z)-d \eta(x, \varphi z) \eta(y)\}
\end{aligned}
$$

Theorem 2.1. The Lee forms $\tilde{\theta}, \tilde{\theta}^{*}$ and $\tilde{\omega}$ of $\mathcal{D}$-homothetic deformation has the relation $\tilde{\theta}(x)=-\theta(x), \tilde{\theta}^{*}(x)=-\theta^{*}(x)$, $\tilde{\omega}(x)=\frac{1}{t} \omega(x)$. 
Proof. By using (2.6) we have

$$
\begin{aligned}
& \tilde{F}\left(e_{i}, e_{i}, x\right)=t F\left(e_{i}, e_{i}, x\right)+\frac{t(t+1)}{2} d \eta\left(\varphi e_{i}, e_{i}\right) \eta(x), \\
& \tilde{F}\left(\tilde{\varphi} e_{i}, \tilde{\varphi} e_{i}, x\right)=t F\left(\varphi e_{i}, \varphi e_{i}, x\right)+\frac{t(t+1)}{2} d \eta\left(\varphi e_{i}, e_{i}\right) \eta(x), \\
& \tilde{F}\left(e_{i}, \tilde{\varphi} e_{i}, x\right)=t F\left(e_{i}, \varphi e_{i}, x\right), \\
& \tilde{F}\left(\tilde{\varphi} e_{i}, e_{i}, x\right)=t F\left(\varphi e_{i}, e_{i}, x\right) .
\end{aligned}
$$

Therefore, by direct computations we have

$$
\begin{aligned}
\tilde{\theta}(x) & =\tilde{g}^{i j} \tilde{F}\left(e_{i}, e_{j}, x\right) \\
& =\sum_{i=1}^{n}-\frac{1}{t} \tilde{F}\left(e_{i}, e_{i}, x\right)+\sum_{i=n+1}^{2 n} \frac{1}{t} \tilde{F}\left(e_{i}, e_{i}, x\right) \\
& =\sum_{i=1}^{n}-\frac{1}{t} \tilde{F}\left(e_{i}, e_{i}, x\right)+\sum_{i=1}^{n} \frac{1}{t} \tilde{F}\left(\varphi e_{i}, \varphi e_{i}, x\right) \\
& =\sum_{i=1}^{n}-\frac{1}{t}\left[t F\left(e_{i}, e_{i}, x\right)+\frac{t(t+1)}{2} d \eta\left(\varphi e_{i}, e_{i}\right) \eta(x)\right. \\
& \left.-t F\left(\varphi e_{i}, \varphi e_{i}, x\right)-\frac{t(t+1)}{2} d \eta\left(\varphi e_{i}, e_{i}\right) \eta(x)\right] \\
& =\sum_{i=1}^{n}\left(-F\left(e_{i}, e_{i}, x\right)+F\left(\varphi e_{i}, \varphi e_{i}, x\right)\right) \\
& =-\theta(x) .
\end{aligned}
$$

and

$$
\begin{aligned}
\tilde{\theta}^{*}(x) & =\tilde{g}^{i j} \tilde{F}\left(e_{i}, e_{j}, x\right) \\
& =\sum_{i=1}^{n}-\frac{1}{t} \tilde{F}\left(e_{i}, \varphi e_{i}, x\right)+\sum_{i=n+1}^{2 n} \frac{1}{t} \tilde{F}\left(e_{i}, \varphi e_{i}, x\right) \\
& =\sum_{i=1}^{n}-\frac{1}{t} \tilde{F}\left(e_{i}, \varphi e_{i}, x\right)-\sum_{i=1}^{n} \frac{1}{t} \tilde{F}\left(\varphi e_{i}, e_{i}, x\right) \\
& =\sum_{i=1}^{n}-\frac{1}{t}\left[\tilde{F}\left(e_{i}, \varphi e_{i}, x\right)+\tilde{F}\left(\varphi e_{i}, e_{i}, x\right)\right] \\
& =\sum_{i=1}^{n}-F\left(e_{i}, \varphi e_{i}, x\right)-F\left(\varphi e_{i}, e_{i}, x\right) \\
& =-\theta^{*}(x) .
\end{aligned}
$$

With the relation (2.6) we get the equality $\tilde{F}(\xi, \xi, x)=t F(\xi, \xi, x)$. Hence,

$$
\tilde{\omega}(x)=\tilde{F}(\tilde{\xi}, \tilde{\xi}, x)=\frac{1}{t^{2}} \tilde{F}(\xi, \xi, x)=\frac{1}{t^{2}} t F(\xi, \xi, x)=\frac{1}{t} \omega(x) .
$$


Theorem 2.2. [6] Let $(M, \varphi, \xi, \eta, g)$ be the class $\mathcal{F}_{i}(i=0,1,4,5)$. In this case, the following conditions hold:

a) If $M \in \mathcal{F}_{0}$, then $N=[\varphi, \varphi]=d \eta=F=\nabla \varphi=\nabla \eta=\nabla \xi=\theta=\theta^{*}=\omega=0$,

b) If $M \in \mathcal{F}_{1}$, then $N=[\varphi, \varphi]=d \eta=\nabla \eta=\nabla \xi=\theta(\xi)=\theta^{*}(\xi)=\omega=0$,

c) If $M \in \mathcal{F}_{4}$, then $N=[\varphi, \varphi]=d \eta=\theta^{*}=\omega=0$,

d) If $M \in \mathcal{F}_{5}$, then $N=[\varphi, \varphi]=d \eta=\theta=\omega=0$.

Theorem 2.3. Let $(M, \varphi, \xi, \eta, g)$ be the class $\mathcal{F}_{i}(i=0,1,4,5)$. Then, $\mathcal{D}$-homothetic deformation $(M, \tilde{\varphi}, \tilde{\xi}, \tilde{\eta}, \tilde{g})$ of $(M, \varphi, \xi, \eta, g)$ belongs to the class $\mathcal{F}_{i}(i=0,1,4,5)$.

Proof. Let $(M, \varphi, \xi, \eta, g)$ be the class $\mathcal{F}_{i}(i=0,1,4,5)$. From the Theorem (2.2) we know that $d \eta=0$. Then, according to the identity (2.6) we obtain immediately

$$
\tilde{F}(x, y, z)=t F(x, y, z) .
$$

1) If $M$ belongs to the class $\mathcal{F}_{0}$ then, $F=0$ and $d \eta=0$. Hence, $\tilde{F}=0$. This implies that $(M, \tilde{\varphi}, \tilde{\xi}, \tilde{\eta}, \tilde{g})$ belongs to the class $\mathcal{F}_{0}$.

2) If $M$ belongs to the class $\mathcal{F}_{1}$, then, we have $d \eta=0$ and the condition of the class $\mathcal{F}_{1}$ is satisfied. By using this condition and (2.7) we obtain

$$
\begin{aligned}
& \frac{1}{2 n}\left\{\tilde{g}(x, \tilde{\varphi} y) \tilde{\theta}(\tilde{\varphi} z)+\tilde{g}(x, \tilde{\varphi} z) \tilde{\theta}(\tilde{\varphi} y)-\tilde{g}(\tilde{\varphi} x, \tilde{\varphi} y) \tilde{\theta}\left(\tilde{\varphi}^{2} z\right)-\tilde{g}(\tilde{\varphi} x, \tilde{\varphi} z) \tilde{\theta}\left(\tilde{\varphi}^{2} y\right)\right\} \\
& =\frac{1}{2 n}\left\{t g(x, \varphi y) \theta(\varphi z)+\operatorname{tg}(x, \varphi z) \theta(\varphi y)-t g(\varphi x, \varphi y) \theta\left(\varphi^{2} z\right)-\operatorname{tg}(\varphi x, \varphi z) \theta\left(\varphi^{2} y\right)\right\} \\
& =t\left[\frac{1}{2 n}\left\{g(x, \varphi y) \theta(\varphi z)+g(x, \varphi z) \theta(\varphi y)-g(\varphi x, \varphi y) \theta\left(\varphi^{2} z\right)-g(\varphi x, \varphi z) \theta\left(\varphi^{2} y\right)\right\}\right] \\
& =t F(x, y, z)=\tilde{F}(x, y, z) .
\end{aligned}
$$

Therefore, $(M, \tilde{\varphi}, \tilde{\xi}, \tilde{\eta}, \tilde{g})$ is in the class $\mathcal{F}_{1}$.

3) Let $M$ be the class $\mathcal{F}_{4}$. Then, we have $d \eta=0$ and the condition of the class $\mathcal{F}_{4}$ is satisfied. Bearing in mind this condition and (2.7) we obtain the following equality:

$$
\begin{aligned}
& -\frac{1}{2 n} \tilde{\theta}(\tilde{\xi})\{\tilde{\eta}(y) \tilde{g}(\tilde{\varphi} x, \tilde{\varphi} z)+\tilde{\eta}(z) g(\tilde{\varphi} x, \tilde{\varphi} y)\} \\
& =-\frac{1}{2 n}\left(-\frac{1}{t} \theta(\xi)\left\{-t^{2} \eta(y) g(\varphi x, \varphi z)-t^{2} \eta(z) g(\varphi x, \varphi y)\right\}\right) \\
& =t\left[-\frac{1}{2 n} \theta(\xi)\{\eta(y) g(\varphi x, \varphi z)+\eta(z) g(\varphi x, \varphi y)\}\right] \\
& =t F(x, y, z)=\tilde{F}(x, y, z) .
\end{aligned}
$$

Hence, $(M, \tilde{\varphi}, \tilde{\xi}, \tilde{\eta}, \tilde{g})$ is the class $\mathcal{F}_{4}$.

4) Let $M$ be the class $\mathcal{F}_{5}$. Bearing in mind $d \eta=0$, the condition of $\mathcal{F}_{5}$ and $(2.7)$ we get the relation

$$
\begin{aligned}
& -\frac{1}{2 n} \tilde{\theta}^{*}(\tilde{\xi})\{\tilde{\eta}(y) \tilde{g}(\tilde{\varphi} x, z)+\tilde{\eta}(z) g(\tilde{\varphi} x, y)\} \\
& =-\frac{1}{2 n}\left(-\frac{1}{t} \theta^{*}(\xi)\left\{-t^{2} \eta(y) g(\varphi x, z)-t^{2} \eta(z) g(\varphi x, y)\right\}\right) \\
& =t\left[-\frac{1}{2 n} \theta^{*}(\xi)\{\eta(y) g(\varphi x, z)+\eta(z) g(\varphi x, y)\}\right] \\
& =t F(x, y, z)=\tilde{F}(x, y, z) .
\end{aligned}
$$

This means that $(M, \tilde{\varphi}, \tilde{\xi}, \tilde{\eta}, \tilde{g})$ is in the class $\mathcal{F}_{5}$. 


\section{Almost contact $B$-metric Structures on $\mathfrak{g}_{i}$}

5 -dimensional nilpotent Lie algebras are divided into nine classes [3]. $\mathfrak{g}_{i}$ 's are five-dimensional nilpotent Lie algebras with corresponding basis $\left\{e_{1}, \ldots, e_{5}\right\}$ and non-zero brackets in the following:

$$
\begin{aligned}
& \mathfrak{g}_{1}:\left[e_{1}, e_{2}\right]=e_{5},\left[e_{3}, e_{4}\right]=e_{5} \\
& \mathfrak{g}_{2}:\left[e_{1}, e_{2}\right]=e_{3},\left[e_{1}, e_{3}\right]=e_{5},\left[e_{2}, e_{4}\right]=e_{5} \\
& \mathfrak{g}_{3}::\left[e_{1}, e_{2}\right]=e_{3},\left[e_{1}, e_{3}\right]=e_{4},\left[e_{1}, e_{4}\right]=e_{5},\left[e_{2}, e_{3}\right]=e_{5} \\
& \mathfrak{g}_{4}::\left[e_{1}, e_{2}\right]=e_{3},\left[e_{1}, e_{3}\right]=e_{4},\left[e_{1}, e_{4}\right]=e_{5} \\
& \mathfrak{g}_{5}:\left[e_{1}, e_{2}\right]=e_{3},\left[e_{1}, e_{3}\right]=e_{4},\left[e_{2}, e_{3}\right]=e_{5} \\
& \mathfrak{g}_{6}::\left[e_{1}, e_{2}\right]=e_{3},\left[e_{1}, e_{3}\right]=e_{4},\left[e_{2}, e_{3}\right]=e_{5}
\end{aligned}
$$

The rest of the classes $\mathfrak{g}_{7}, \mathfrak{g}_{8}, \mathfrak{g}_{9}$ are abelian. Almost contact metric structures on $\mathfrak{g}_{i}$ were defined and the classification of five-dimensional nilpotent Lie algebras are obtained in [3]. Moreover, almost contact metric structures on 5-dimensional nilpotent Lie algebras are investigated in [9].

Firstly, we investigate the classes of the following almost contact $B$-metric structure with respect to the basis $\left\{e_{1}, \ldots, e_{5}\right\}$ on each $\mathfrak{g}_{i}$.

$$
\begin{aligned}
& g\left(e_{1}, e_{1}\right)=g\left(e_{2}, e_{2}\right)=g\left(e_{5}, e_{5}\right)=-g\left(e_{3}, e_{3}\right)=-g\left(e_{4}, e_{4}\right)=1, \\
& \varphi\left(e_{1}\right)=e_{3}, \varphi\left(e_{2}\right)=e_{4}, \varphi\left(e_{3}\right)=-e_{1}, \varphi\left(e_{4}\right)=-e_{2}, \varphi\left(e_{5}\right)=0, \\
& \xi=e_{5}, \eta=e^{5} .
\end{aligned}
$$

Theorem 3.1. The Lie algebra $\mathfrak{g}_{1}$ belongs to the class $\mathcal{F}_{8} \oplus \mathcal{F}_{10}$.

Proof. By using the non-zero brackets $\left[e_{1}, e_{2}\right]=e_{5},\left[e_{3}, e_{4}\right]=e_{5}$ and Kozsul's formula, the covariant derivatives of the non-zero basic elements are given by

$$
\begin{aligned}
& \nabla_{e_{1}} e_{2}=\frac{1}{2} e_{5}, \nabla_{e_{1}} e_{5}=-\frac{1}{2} e_{2}, \nabla_{e_{2}} e_{1}=-\frac{1}{2} e_{5}, \nabla_{e_{2}} e_{5}=\frac{1}{2} e_{1}, \\
& \nabla_{e_{3}} e_{4}=\frac{1}{2} e_{5}, \nabla_{e_{3}} e_{5}=\frac{1}{2} e_{4}, \nabla_{e_{4}} e_{3}=-\frac{1}{2} e_{5}, \nabla_{e_{4}} e_{5}=-\frac{1}{2} e_{3}, \\
& \nabla_{e_{5}} e_{1}=-\frac{1}{2} e_{2}, \nabla_{e_{5}} e_{2}=\frac{1}{2} e_{1}, \nabla_{e_{5}} e_{3}=\frac{1}{2} e_{4}, \nabla_{e_{5}} e_{4}=-\frac{1}{2} e_{3} .
\end{aligned}
$$

For $i=1, \ldots, 5$, we get $F\left(e_{i}, e_{i}, x\right)=0$ and $F\left(e_{i}, \varphi e_{i}, x\right)=0$. Thus, by $(2.3)$ it is easy to see that $\theta(x)=\theta^{*}(x)=0$. Moreover, we get the following non-zero components $F\left(e_{i}, e_{j}, e_{k}\right)=F_{i j k}$ of the structure tensor:

$$
\begin{aligned}
& F_{145}=F_{415}=F_{154}=F_{451}=-\frac{1}{2}, \\
& F_{514}=F_{541}=-1, \\
& F_{235}=F_{325}=F_{253}=F_{352}=\frac{1}{2}, \\
& F_{523}=F_{532}=1 .
\end{aligned}
$$

Then, we establish the following form of $F$ for arbitrary vectors $x, y, z$ :

$$
\begin{aligned}
F(x, y, z)= & F\left(\sum_{i} x_{i} e_{i}, \sum_{j} y_{j} e_{j}, \sum_{k} z_{k} e_{k}\right) \\
= & \sum_{i, j, k} x_{i} y_{j} z_{k} F\left(e_{i}, e_{j}, e_{k}\right) \\
= & -\frac{1}{2} x_{1} y_{4} z_{5}-\frac{1}{2} x_{4} y_{1} z_{5}-\frac{1}{2} x_{1} y_{5} z_{4}-\frac{1}{2} x_{4} y_{5} z_{1}-x_{5} y_{1} z_{4}-x_{5} y_{4} z_{1} \\
& +\frac{1}{2} x_{2} y_{3} z_{5}+\frac{1}{2} x_{3} y_{2} z_{5}+\frac{1}{2} x_{2} y_{5} z_{3}+\frac{1}{2} x_{3} y_{5} z_{2}+x_{5} y_{2} z_{3}+x_{5} y_{3} z_{2} .
\end{aligned}
$$

The latter equality implies that $F$ is represented in the form $F(x, y, z)=F_{8}(x, y, z)+F_{10}(x, y, z)$. Therefore, we prove that $\mathfrak{g}_{1}$ belongs to the class $\mathcal{F}_{8} \oplus \mathcal{F}_{10}$. 
Theorem 3.2. The Lie algebra $\mathfrak{g}_{2}$ belongs to the class $\mathcal{F}_{2} \oplus \mathcal{F}_{8} \oplus \mathcal{F}_{10}$.

Proof. By using the relations $\left[e_{1}, e_{2}\right]=e_{3},\left[e_{1}, e_{3}\right]=e_{5},\left[e_{2}, e_{4}\right]=e_{5}$ and Kozsul's formula we get

$$
\begin{aligned}
& \nabla_{e_{1}} e_{2}=\frac{1}{2} e_{3}, \nabla_{e_{1}} e_{3}=\frac{1}{2} e_{2}+\frac{1}{2} e_{5}, \nabla_{e_{1}} e_{5}=\frac{1}{2} e_{3}, \nabla_{e_{2}} e_{1}=-\frac{1}{2} e_{3}, \\
& \nabla_{e_{2}} e_{3}=-\frac{1}{2} e_{1}, \nabla_{e_{2}} e_{4}=\frac{1}{2} e_{5}, \nabla_{e_{2}} e_{5}=\frac{1}{2} e_{4}, \nabla_{e_{3}} e_{1}=\frac{1}{2} e_{2}-\frac{1}{2} e_{5}, \\
& \nabla_{e_{3}} e_{2}=-\frac{1}{2} e_{1}, \nabla_{e_{3}} e_{5}=\frac{1}{2} e_{1}, \nabla_{e_{4}} e_{2}=-\frac{1}{2} e_{5}, \nabla_{e_{4}} e_{5}=\frac{1}{2} e_{2}, \\
& \nabla_{e_{5}} e_{1}=\frac{1}{2} e_{3}, \nabla_{e_{5}} e_{2}=\frac{1}{2} e_{4}, \nabla_{e_{5}} e_{3}=\frac{1}{2} e_{1}, \nabla_{e_{5}} e_{4}=\frac{1}{2} e_{2} .
\end{aligned}
$$

By (2.3) we have $\theta(\xi)=\theta^{*}(\xi)=0$ and $\theta(x)=x_{2}, \theta^{*}(x)=x_{4}$ for any $x=\sum_{i=1}^{5} x_{i} e_{i}$.

The components of the structure tensor $F\left(e_{i}, e_{j}, e_{k}\right)=F_{i j k}$ can be found as

$$
\begin{aligned}
& F_{112}=F_{121}=F_{134}=F_{143}=F_{341}=F_{314}=\frac{1}{2}, \\
& F_{115}=F_{225}=F_{335}=F_{445}=F_{151}=F_{252}=F_{353}=F_{454}=\frac{1}{2}, \\
& F_{332}=F_{323}=-\frac{1}{2}, \\
& F_{511}=F_{522}=F_{533}=F_{544}=1 .
\end{aligned}
$$

For any $x, y, z$, the tensor $F$ can be written in the following way:

$$
\begin{aligned}
F(x, y, z)= & F\left(\sum_{i} x_{i} e_{i}, \sum_{j} y_{j} e_{j}, \sum_{k} z_{k} e_{k}\right) \\
= & \sum_{i, j, k} x_{i} y_{j} z_{k} F\left(e_{i}, e_{j}, e_{k}\right) \\
= & \frac{1}{2} x_{1} y_{1} z_{2}+\frac{1}{2} x_{1} y_{2} z_{1}+\frac{1}{2} x_{1} y_{3} z_{4}+\frac{1}{2} x_{1} y_{4} z_{3}+\frac{1}{2} x_{3} y_{4} z_{1}+\frac{1}{2} x_{3} y_{1} z_{4} \\
& +\frac{1}{2} x_{1} y_{1} z_{5}+\frac{1}{2} x_{2} y_{2} z_{5}+\frac{1}{2} x_{3} y_{3} z_{5}+\frac{1}{2} x_{4} y_{4} z_{5}+\frac{1}{2} x_{1} y_{5} z_{1}+\frac{1}{2} x_{2} y_{5} z_{2} \\
& +\frac{1}{2} x_{3} y_{5} z_{3}+\frac{1}{2} x_{4} y_{5} z_{4}-\frac{1}{2} x_{3} y_{3} z_{2}-\frac{1}{2} x_{3} y_{2} z_{3}+x_{5} y_{1} z_{1}+x_{5} y_{2} z_{2} \\
& +x_{5} y_{3} z_{3}+x_{5} y_{4} z_{4} .
\end{aligned}
$$

Since

$$
\begin{aligned}
F_{8}(x, y, z) & =\frac{1}{2} x_{1} y_{1} z_{5}+\frac{1}{2} x_{2} y_{2} z_{5}+\frac{1}{2} x_{3} y_{3} z_{5}+\frac{1}{2} x_{4} y_{4} z_{5}+\frac{1}{2} x_{1} y_{5} z_{1} \\
+ & \frac{1}{2} x_{2} y_{5} z_{2}+\frac{1}{2} x_{3} y_{5} z_{3}+\frac{1}{2} x_{4} y_{5} z_{4} \\
F_{10}(x, y, z) & =x_{5} y_{1} z_{1}+x_{5} y_{2} z_{2}+x_{5} y_{3} z_{3}+x_{5} y_{4} z_{4}
\end{aligned}
$$

and the other terms are in the class $\mathcal{F}_{2}$, the tensor $F$ can be written as $F(x, y, z)=F_{2}(x, y, z)+F_{8}(x, y, z)+$ $F_{10}(x, y, z)$. This means that $F \in \mathcal{F}_{2} \oplus \mathcal{F}_{8} \oplus \mathcal{F}_{10}$. Hence, the Lie algebra $\mathfrak{g}_{2}$ is in the class $\mathcal{F}_{2} \oplus \mathcal{F}_{8} \oplus \mathcal{F}_{10}$.

Theorem 3.3. The Lie algebra $\mathfrak{g}_{3}$ belongs to the class $\mathcal{F}_{1} \oplus \mathcal{F}_{2} \oplus \mathcal{F}_{3} \oplus \mathcal{F}_{8} \oplus \mathcal{F}_{10}$. 
Proof. Using $\left[e_{1}, e_{2}\right]=e_{3},\left[e_{1}, e_{3}\right]=e_{4},\left[e_{1}, e_{4}\right]=e_{5},\left[e_{2}, e_{3}\right]=e_{5}$, we compute the non-zero components of the Levi-Civita connection $\nabla$ with respect to $g$ as follows:

$$
\begin{aligned}
& \nabla_{e_{1}} e_{2}=\frac{1}{2} e_{3}, \nabla_{e_{1}} e_{3}=\frac{1}{2} e_{2}+\frac{1}{2} e_{4}, \nabla_{e_{1}} e_{4}=-\frac{1}{2} e_{3}+\frac{1}{2} e_{5}, \nabla_{e_{1}} e_{5}=\frac{1}{2} e_{4}, \\
& \nabla_{e_{2}} e_{1}=-\frac{1}{2} e_{3}, \nabla_{e_{2}} e_{3}=-\frac{1}{2} e_{1}+\frac{1}{2} e_{5}, \nabla_{e_{2}} e_{5}=\frac{1}{2} e_{3}, \nabla_{e_{3}} e_{1}=\frac{1}{2} e_{2}-\frac{1}{2} e_{4}, \\
& \nabla_{e_{3}} e_{2}=-\frac{1}{2} e_{1}-\frac{1}{2} e_{5}, \nabla_{e_{3}} e_{4}=-\frac{1}{2} e_{1}, \nabla_{e_{3}} e_{5}=\frac{1}{2} e_{2}, \nabla_{e_{4}} e_{1}=-\frac{1}{2} e_{3}-\frac{1}{2} e_{5}, \\
& \nabla_{e_{4}} e_{3}=-\frac{1}{2} e_{1}, \nabla_{e_{4}} e_{5}=\frac{1}{2} e_{1}, \nabla_{e_{5}} e_{1}=\frac{1}{2} e_{4}, \nabla_{e_{5}} e_{2}=\frac{1}{2} e_{3}, \nabla_{e_{5}} e_{3}=\frac{1}{2} e_{2}, \\
& \nabla_{e_{5}} e_{4}=\frac{1}{2} e_{1} .
\end{aligned}
$$

By (2.3) it is easy to verify that $\theta(\xi)=\theta^{*}(\xi)=0$ and $\theta(x)=x_{2}, \theta^{*}(x)=x_{4}$ for any $x=\sum_{i=1}^{5} x_{i} e_{i}$. By virtue of the latter equalities we establish the basic components $F\left(e_{i}, e_{j}, e_{k}\right)=F_{i j k}$ of $F$ in the following:

$$
\begin{aligned}
& F_{112}=F_{121}=F_{123}=F_{132}=F_{125}=F_{152}=F_{134}=F_{143}=\frac{1}{2}, \\
& F_{215}=F_{251}=F_{314}=F_{341}=F_{345}=F_{354}=F_{435}=F_{453}=\frac{1}{2}, \\
& F_{114}=F_{141}=F_{312}=F_{321}=F_{323}=F_{332}=F_{334}=F_{343}=-\frac{1}{2}, \\
& F_{211}=F_{233}=F_{433}=F_{411}=-1, \\
& F_{512}=F_{521}=F_{534}=F_{543}=1 .
\end{aligned}
$$

Then, we have

$$
\begin{aligned}
F(x, y, z)= & F\left(\sum_{i} x_{i} e_{i}, \sum_{j} y_{j} e_{j}, \sum_{k} z_{k} e_{k}\right) \\
= & \sum_{i, j, k} x_{i} y_{j} z_{k} F\left(e_{i}, e_{j}, e_{k}\right) \\
= & \frac{1}{2} x_{1} y_{1} z_{2}+\frac{1}{2} x_{1} y_{2} z_{1}+\frac{1}{2} x_{1} y_{2} z_{3}+\frac{1}{2} x_{1} y_{3} z_{2}+\frac{1}{2} x_{1} y_{2} z_{5} \\
& +\frac{1}{2} x_{1} y_{5} z_{2}+\frac{1}{2} x_{1} y_{3} z_{4}+\frac{1}{2} x_{1} y_{4} z_{3}+\frac{1}{2} x_{2} y_{1} z_{5}+\frac{1}{2} x_{2} y_{5} z_{1} \\
& +\frac{1}{2} x_{3} y_{1} z_{4}+\frac{1}{2} x_{3} y_{4} z_{1}+\frac{1}{2} x_{3} y_{4} z_{5}+\frac{1}{2} x_{3} y_{5} z_{4}+\frac{1}{2} x_{4} y_{3} z_{5} \\
& +\frac{1}{2} x_{4} y_{5} z_{3}-\frac{1}{2} x_{1} y_{1} z_{4}-\frac{1}{2} x_{1} y_{4} z_{1}-\frac{1}{2} x_{3} y_{1} z_{2}-\frac{1}{2} x_{3} y_{2} z_{1} \\
& -\frac{1}{2} x_{3} y_{2} z_{3}-\frac{1}{2} x_{3} y_{3} z_{2}-\frac{1}{2} x_{3} y_{3} z_{4}-\frac{1}{2} x_{3} y_{4} z_{3}-x_{2} y_{1} z_{1} \\
& -x_{2} y_{3} z_{3}-x_{4} y_{3} z_{3}-x_{4} y_{1} z_{1}+x_{5} y_{1} z_{2}+x_{5} y_{2} z_{1}+x_{5} y_{3} z_{4}+x_{5} y_{4} z_{3}
\end{aligned}
$$

where

$$
\begin{gathered}
F_{8}(x, y, z)=\frac{1}{2} x_{1} y_{2} z_{5}+\frac{1}{2} x_{2} y_{1} z_{5}+\frac{1}{2} x_{3} y_{4} z_{5}+\frac{1}{2} x_{4} y_{3} z_{5}+\frac{1}{2} x_{1} y_{5} z_{2} \\
+\frac{1}{2} x_{2} y_{5} z_{1}+\frac{1}{2} x_{3} y_{5} z_{4}+\frac{1}{2} x_{4} y_{5} z_{3}
\end{gathered}
$$

$F_{10}(x, y, z)=x_{5} y_{1} z_{2}+x_{5} y_{2} z_{1}+x_{5} y_{3} z_{4}+x_{5} y_{4} z_{3}$ 
and the other terms are in the space $W_{1}=F_{1}(x, y, z)+F_{2}(x, y, z)+F_{3}(x, y, z)$, the tensor $F$ is $F(x, y, z)=$ $W_{1}+F_{8}(x, y, z)+F_{10}(x, y, z)$. That is, $F \in \mathcal{F}_{1} \oplus \mathcal{F}_{2} \oplus \mathcal{F}_{3} \oplus \mathcal{F}_{8} \oplus \mathcal{F}_{10}$. Therefore, the lie algebra $\mathfrak{g}_{3}$ is in the class $\mathcal{F}_{1} \oplus \mathcal{F}_{2} \oplus \mathcal{F}_{3} \oplus \mathcal{F}_{8} \oplus \mathcal{F}_{10}$.

Theorem 3.4. The Lie algebras $\mathfrak{g}_{4}$ belongs to the class $\mathcal{F}_{2} \oplus \mathcal{F}_{3} \oplus \mathcal{F}_{8} \oplus \mathcal{F}_{10}$.

Proof. With the aid of the relations given in $\mathfrak{g}_{4}$, the basic components of the Levi-Civita connection $\nabla$ can be found as

$$
\begin{aligned}
& \nabla_{e_{1}} e_{2}=\frac{1}{2} e_{3}, \nabla_{e_{1}} e_{3}=\frac{1}{2} e_{2}+\frac{1}{2} e_{4}, \nabla_{e_{1}} e_{4}=-\frac{1}{2} e_{3}+\frac{1}{2} e_{5}, \nabla_{e_{1}} e_{5}=\frac{1}{2} e_{4}, \\
& \nabla_{e_{2}} e_{1}=-\frac{1}{2} e_{3}, \nabla_{e_{2}} e_{3}=-\frac{1}{2} e_{1}, \nabla_{e_{3}} e_{1}=\frac{1}{2} e_{2}-\frac{1}{2} e_{4}, \nabla_{e_{3}} e_{2}=-\frac{1}{2} e_{1}, \\
& \nabla_{e_{3}} e_{4}=-\frac{1}{2} e_{1}, \nabla_{e_{4}} e_{1}=-\frac{1}{2} e_{3}-\frac{1}{2} e_{5}, \nabla_{e_{4}} e_{3}=-\frac{1}{2} e_{1}, \nabla_{e_{4}} e_{5}=\frac{1}{2} e_{1}, \\
& \nabla_{e_{5}} e_{1}=\frac{1}{2} e_{4}, \nabla_{e_{5}} e_{4}=\frac{1}{2} e_{1} .
\end{aligned}
$$

By (2.3) it can be easily check that $\theta(\xi)=\theta^{*}(\xi)=0$ and $\theta(x)=\theta^{*}(x)=0$ for any $x=\sum_{i=1}^{5} x_{i} e_{i}$. The components $F\left(e_{i}, e_{j}, e_{k}\right)=F_{i j k}$ of $F$ are given by

$$
\begin{aligned}
& F_{112}=F_{121}=F_{123}=F_{132}=F_{125}=F_{152}=F_{134}=F_{143}=\frac{1}{2}, \\
& F_{314}=F_{341}=F_{435}=F_{453}=F_{512}=F_{521}=F_{534}=F_{543}=\frac{1}{2}, \\
& F_{114}=F_{141}=F_{312}=F_{321}=F_{323}=F_{332}=F_{334}=F_{343}=-\frac{1}{2}, \\
& F_{411}=F_{433}=-1 .
\end{aligned}
$$

Hence, we obtain

$$
\begin{aligned}
F(x, y, z)= & F\left(\sum_{i} x_{i} e_{i}, \sum_{j} y_{j} e_{j}, \sum_{k} z_{k} e_{k}\right) \\
= & \sum_{i, j, k} x_{i} y_{j} z_{k} F\left(e_{i}, e_{j}, e_{k}\right) \\
= & \frac{1}{2} x_{1} y_{1} z_{2}+\frac{1}{2} x_{1} y_{2} z_{1}+\frac{1}{2} x_{1} y_{2} z_{3}+\frac{1}{2} x_{1} y_{3} z_{2}+\frac{1}{2} x_{1} y_{2} z_{5} \\
& +\frac{1}{2} x_{1} y_{5} z_{2}+\frac{1}{2} x_{1} y_{3} z_{4}+\frac{1}{2} x_{1} y_{4} z_{3}-\frac{1}{2} x_{3} y_{2} z_{3}-\frac{1}{2} x_{3} y_{3} z_{2} \\
& +\frac{1}{2} x_{4} y_{3} z_{5}+\frac{1}{2} x_{4} y_{5} z_{3}+\frac{1}{2} x_{5} y_{1} z_{2}+\frac{1}{2} x_{5} y_{2} z_{1}+\frac{1}{2} x_{5} y_{3} z_{4} \\
& +\frac{1}{2} x_{5} y_{4} z_{3}-\frac{1}{2} x_{1} y_{1} z_{4}-\frac{1}{2} x_{1} y_{4} z_{1}-\frac{1}{2} x_{3} y_{1} z_{2}-\frac{1}{2} x_{3} y_{2} z_{1} \\
& +\frac{1}{2} x_{3} y_{1} z_{4}+\frac{1}{2} x_{3} y_{4} z_{1}-\frac{1}{2} x_{3} y_{3} z_{4}-\frac{1}{2} x_{3} y_{4} z_{3}-x_{4} y_{1} z_{1}-x_{4} y_{3} z_{3}
\end{aligned}
$$

where

$$
\begin{aligned}
& F_{8}(x, y, z)=\frac{1}{2} x_{1} y_{2} z_{5}+\frac{1}{2} x_{3} y_{4} z_{5}+\frac{1}{2} x_{1} y_{5} z_{2}+\frac{1}{2} x_{4} y_{5} z_{3}, \\
& F_{10}(x, y, z)=\frac{1}{2} x_{5} y_{1} z_{2}+\frac{1}{2} x_{5} y_{2} z_{1}+\frac{1}{2} x_{5} y_{3} z_{4}+\frac{1}{2} x_{5} y_{4} z_{3} .
\end{aligned}
$$

Since $F(x, y, z)=F_{2}(x, y, z)+F_{3}(x, y, z)+F_{8}(x, y, z)+F_{10}(x, y, z)$, the tensor $F$ is in the class $\mathcal{F}_{2} \oplus \mathcal{F}_{3} \oplus \mathcal{F}_{8} \oplus$ $\mathcal{F}_{10}$. Then, the Lie algebra $\mathfrak{g}_{4}$ belongs to the class $\mathcal{F}_{2} \oplus \mathcal{F}_{3} \oplus \mathcal{F}_{8} \oplus \mathcal{F}_{10}$. 
Theorem 3.5. The class of the Lie algebra $\mathfrak{g}_{5}$ is $\mathcal{F}_{1} \oplus \mathcal{F}_{3} \oplus \mathcal{F}_{8} \oplus \mathcal{F}_{10}$.

Proof. In similar way, the basic terms of the Levi-Civita connection $\nabla$ can be calculated as

$$
\begin{aligned}
& \nabla_{e_{1}} e_{2}=\frac{1}{2} e_{4}, \nabla_{e_{1}} e_{3}=\frac{1}{2} e_{5}, \nabla_{e_{1}} e_{4}=\frac{1}{2} e_{2}, \nabla_{e_{1}} e_{5}=\frac{1}{2} e_{3}, \\
& \nabla_{e_{2}} e_{1}=-\frac{1}{2} e_{4}, \nabla_{e_{2}} e_{4}=-\frac{1}{2} e_{1}, \nabla_{e_{3}} e_{1}=-\frac{1}{2} e_{5}, \nabla_{e_{3}} e_{5}=\frac{1}{2} e_{1}, \\
& \nabla_{e_{4}} e_{1}=\frac{1}{2} e_{2}, \nabla_{e_{4}} e_{2}=-\frac{1}{2} e_{1}, \nabla_{e_{5}} e_{1}=\frac{1}{2} e_{3}, \nabla_{e_{5}} e_{3}=\frac{1}{2} e_{1} .
\end{aligned}
$$

By direct computation we obtain $\theta(\xi)=\theta^{*}(\xi)=0$ and $\theta(x)=-x_{1}, \theta^{*}(x)=-x_{3}$ for any $x$. We get the basic components $F\left(e_{i}, e_{j}, e_{k}\right)=F_{i j k}$ as follows:

$$
\begin{aligned}
& F_{115}=F_{151}=F_{335}=F_{353}=F_{414}=F_{441}=\frac{1}{2} \\
& F_{212}=F_{221}=F_{234}=F_{243}=F_{423}=F_{432}=-\frac{1}{2} \\
& F_{122}=F_{144}=F_{511}=F_{533}=1
\end{aligned}
$$

Therefore, the tensor $F$ is written as

$$
\begin{aligned}
F(x, y, z)= & F\left(\sum_{i} x_{i} e_{i}, \sum_{j} y_{j} e_{j}, \sum_{k} z_{k} e_{k}\right) \\
= & \sum_{i, j, k} x_{i} y_{j} z_{k} F\left(e_{i}, e_{j}, e_{k}\right) \\
= & \frac{1}{2} x_{1} y_{1} z_{5}+\frac{1}{2} x_{1} y_{5} z_{1}+\frac{1}{2} x_{3} y_{3} z_{5}+\frac{1}{2} x_{3} y_{5} z_{3}+\frac{1}{2} x_{4} y_{1} z_{4} \\
& +\frac{1}{2} x_{4} y_{4} z_{1}-\frac{1}{2} x_{2} y_{1} z_{2}-\frac{1}{2} x_{2} y_{2} z_{1}-\frac{1}{2} x_{2} y_{3} z_{4}-\frac{1}{2} x_{2} y_{4} z_{3} \\
& -\frac{1}{2} x_{4} y_{2} z_{3}-\frac{1}{2} x_{4} y_{3} z_{2}+x_{1} y_{2} z_{2}+x_{1} y_{4} z_{4}+x_{5} y_{1} z_{1}+x_{5} y_{3} z_{3} .
\end{aligned}
$$

It can be easily see that $F$ is in the class $\mathcal{F}_{1} \oplus \mathcal{F}_{3} \oplus \mathcal{F}_{8} \oplus \mathcal{F}_{10}$. Namely, the Lie algebra $\mathfrak{g}_{5}$ belongs to $\mathcal{F}_{1} \oplus \mathcal{F}_{3} \oplus$ $\mathcal{F}_{8} \oplus \mathcal{F}_{10}$.

Theorem 3.6. The Lie algebra $\mathfrak{g}_{6}$ belongs to the class $\mathcal{F}_{1} \oplus \mathcal{F}_{2} \oplus \mathcal{F}_{3} \oplus \mathcal{F}_{8} \oplus \mathcal{F}_{10}$.

Proof. Similarly, the basic components of $\nabla$ are computed as follows:

$$
\begin{aligned}
& \nabla_{e_{1}} e_{2}=\frac{1}{2} e_{3}, \nabla_{e_{1}} e_{3}=\frac{1}{2} e_{2}+\frac{1}{2} e_{4}, \nabla_{e_{1}} e_{4}=-\frac{1}{2} e_{3}, \nabla_{e_{2}} e_{1}=-\frac{1}{2} e_{3} \\
& \nabla_{e_{2}} e_{3}=-\frac{1}{2} e_{1}+\frac{1}{2} e_{5}, \nabla_{e_{2}} e_{5}=\frac{1}{2} e_{3}, \nabla_{e_{3}} e_{1}=\frac{1}{2} e_{2}-\frac{1}{2} e_{4}, \nabla_{e_{3}} e_{2}=-\frac{1}{2} e_{1}-\frac{1}{2} e_{5}, \\
& \nabla_{e_{3}} e_{4}=-\frac{1}{2} e_{1}, \nabla_{e_{3}} e_{5}=\frac{1}{2} e_{2}, \nabla_{e_{4}} e_{1}=-\frac{1}{2} e_{3}, \nabla_{e_{4}} e_{3}=-\frac{1}{2} e_{1}, \nabla_{e_{5}} e_{2}=\frac{1}{2} e_{3}, \nabla_{e_{5}} e_{3}=\frac{1}{2} e_{2} .
\end{aligned}
$$

Then, using the equality (2.3) we get the identities $\theta(\xi)=\theta^{*}(\xi)=0, \theta(x)=x_{2}$ and $\theta^{*}(x)=x_{4}$ for any $x$. The basic components $F_{i j k}$ are

$$
\begin{aligned}
& F_{112}=F_{121}=F_{123}=F_{132}=F_{215}=F_{251}=F_{134}=F_{143}=\frac{1}{2}, \\
& F_{314}=F_{341}=F_{512}=F_{521}=F_{534}=F_{543}=F_{345}=F_{354}=\frac{1}{2} \\
& F_{114}=F_{141}=F_{312}=F_{321}=F_{323}=F_{332}=F_{334}=F_{343}=-\frac{1}{2}, \\
& F_{211}=F_{233}=F_{433}=F_{411}=-1 .
\end{aligned}
$$


Therefore, the latter equalities imply that $F$ is represented in the form

$$
\begin{aligned}
F(x, y, z)= & F\left(\sum_{i} x_{i} e_{i}, \sum_{j} y_{j} e_{j}, \sum_{k} z_{k} e_{k}\right) \\
= & \sum_{i, j, k} x_{i} y_{j} z_{k} F\left(e_{i}, e_{j}, e_{k}\right) \\
= & \frac{1}{2} x_{1} y_{1} z_{2}+\frac{1}{2} x_{1} y_{2} z_{1}+\frac{1}{2} x_{1} y_{2} z_{3}+\frac{1}{2} x_{1} y_{3} z_{2}+\frac{1}{2} x_{2} y_{1} z_{5}+\frac{1}{2} x_{2} y_{5} z_{1} \\
& +\frac{1}{2} x_{1} y_{3} z_{4}+\frac{1}{2} x_{1} y_{4} z_{3}+\frac{1}{2} x_{3} y_{1} z_{4}+\frac{1}{2} x_{3} y_{4} z_{1}+\frac{1}{2} x_{5} y_{1} z_{2}+\frac{1}{2} x_{5} y_{2} z_{1} \\
& +\frac{1}{2} x_{5} y_{3} z_{4}+\frac{1}{2} x_{5} y_{4} z_{3}-\frac{1}{2} x_{1} y_{1} z_{4}-\frac{1}{2} x_{1} y_{4} z_{1}-\frac{1}{2} x_{3} y_{1} z_{2}-\frac{1}{2} x_{3} y_{2} z_{1} \\
& -\frac{1}{2} x_{3} y_{2} z_{3}-\frac{1}{2} x_{3} y_{3} z_{2}-\frac{1}{2} x_{3} y_{3} z_{4}-\frac{1}{2} x_{3} y_{4} z_{3}+\frac{1}{2} x_{3} y_{4} z_{5}+\frac{1}{2} x_{3} y_{5} z_{4} \\
& -x_{2} y_{1} z_{1}-x_{2} y_{3} z_{3}-x_{4} y_{3} z_{3}-x_{4} y_{1} z_{1} .
\end{aligned}
$$

It is easily checked that $F \in \mathcal{F}_{1} \oplus \mathcal{F}_{2} \oplus \mathcal{F}_{3} \oplus \mathcal{F}_{8} \oplus \mathcal{F}_{10}$. That is, the Lie algebra $\mathfrak{g}_{6}$ is in the class $\mathcal{F}_{1} \oplus \mathcal{F}_{2} \oplus \mathcal{F}_{3} \oplus$ $\mathcal{F}_{8} \oplus \mathcal{F}_{10}$.

Theorem 3.7. The vector field $\xi$ defined on the Lie algebras $\mathfrak{g}_{i}$ for $i=1, \ldots, 6$ is a Killing vector field.

Proof. Let $x=\sum_{i} x_{i} e_{i}$ and $y=\sum_{j} y_{j} e_{j}$. Now, we will show that the following condition is satisfied for every $\mathfrak{g}_{i}$ :

$$
g\left(\nabla_{x} \xi, y\right)=-g\left(\nabla_{y} \xi, x\right)
$$

For the Lie algebra $\mathfrak{g}_{1}$, we obtain that

$$
\begin{aligned}
g\left(\nabla_{x} \xi, y\right) & =\sum_{i, j} x_{i} y_{j} g\left(\nabla_{e_{i}} \xi, e_{j}\right) \\
& =-x_{1} y_{2} \frac{1}{2}+x_{2} y_{1} \frac{1}{2}-x_{3} y_{4} \frac{1}{2}+x_{4} y_{3} \frac{1}{2} \\
g\left(\nabla_{y} \xi, x\right) & =\sum_{i, j} y_{j} x_{i} g\left(\nabla_{e_{j}} \xi, e_{i}\right) \\
& =-y_{1} x_{2} \frac{1}{2}+y_{2} x_{1} \frac{1}{2}-y_{3} x_{4} \frac{1}{2}+y_{4} x_{3} \frac{1}{2} .
\end{aligned}
$$

Thus, the vector field $\xi$ is a Killing vector field for $\mathfrak{g}_{1}$.

For the Lie algebra $\mathfrak{g}_{2}$, we get

$$
g\left(\nabla_{x} \xi, y\right)=-x_{1} y_{3} \frac{1}{2}-x_{2} y_{4} \frac{1}{2}+x_{3} y_{1} \frac{1}{2}+x_{4} y_{2} \frac{1}{2}=g\left(\nabla_{y} \xi, x\right)
$$

Therefore, $\xi$ is a Killing vector field for $\mathfrak{g}_{2}$. In similar way, it can be easily shown that the vector field $\xi$ is a Killing vector field for the Lie algebras $\mathfrak{g}_{3}, \mathfrak{g}_{4}, \mathfrak{g}_{5}$ and $\mathfrak{g}_{6}$.

Corollary 3.1. 5 -dimensional nilpotent Lie algebras $\mathfrak{g}_{i}$ for $i=1, \ldots, 6$ are $K$-contact $B$-metric manifolds.

By the straightforward computations we establish the following theorem:

Theorem 3.8. The Ricci curvatures and scalar curvatures of 5 -dimensional nilpotent Lie algebras $\mathfrak{g}_{i}$ for $i=1, \ldots, 6$ are calculated as 


\begin{tabular}{|c|c|c|}
\hline & Ricci Curvature R $(x, y)$ & Scalar Curvatures \\
\hline $\mathfrak{g}_{1}$ & $-\frac{1}{2} x^{1} y^{1}-\frac{1}{2} x^{2} y^{2}+\frac{1}{2} x^{3} y^{3}+\frac{1}{2} x^{4} y^{4}+x^{5} y^{5}$ & -1 \\
\hline $\mathfrak{g}_{2}$ & $x^{1} y^{1}+x^{2} y^{2}-\frac{1}{2} x^{4} y^{4}-x^{5} y^{5}$ & $\frac{3}{2}$ \\
\hline $\mathfrak{g}_{3}$ & $-\frac{1}{2} x^{1} y^{1}-\frac{1}{2} x^{1} y^{5}+x^{2} y^{2}+\frac{1}{2} x^{3} y^{3}-x^{4} y^{4}-x^{5} y^{5}$ & $\frac{1}{2}$ \\
\hline $\mathfrak{g}_{4}$ & $\frac{1}{2} x^{1} y^{1}+\frac{1}{2} x^{2} y^{2}+x^{3} y^{3}-\frac{1}{2} x^{4} y^{4}+\frac{1}{2} x^{5} y^{5}$ & 1 \\
\hline $\mathfrak{g}_{5}$ & $x^{1} y^{1}+\frac{1}{2} x^{2} y^{2}-\frac{1}{2} x^{3} y^{3}+\frac{1}{2} x^{4} y^{4}-\frac{1}{2} x^{5} y^{5}$ & 1 \\
\hline $\mathfrak{g}_{6}$ & $x^{2} y^{2}+\frac{1}{2} x^{3} y^{3}-\frac{1}{2} x^{4} y^{4}-\frac{1}{2} x^{5} y^{5}$ & $\frac{1}{2}$ \\
\hline
\end{tabular}

\section{References}

[1] Bulut, Ş.: D-homothetic deformation on almost contact B-metric manifolds. J. of Geo. 110(23), (2019).

[2] Chinea, D., Gonzalez, C.: A classification of almost contact metric manifolds. An.Sti.Univ."Al.I.Cuza"Iasi. 30, 75-79 (1984).

[3] Dixmier, J.: Sur les representations unitaires des groupes de Lie nilpotentes III. Canadian Journal of Mathematics. 10, 321-348 (1958).

[4] Ganchev, G., Borisov, A.: Note on almost contact manifolds with Norden metric. Compt. Rend. Acad. Bulg. Sci. 39, 31-34 (1986).

[5] Ganchev, G., Mihova, V., Gribachev, K.: Almost contact manifolds with B-metric. Mathematica Balkanica. 7, 261-276 (1993).

[6] Manev, M.: Curvature properties on some classes of almost contact manifolds with B-metric. Compt. Rend. Acad. Bulg. Sci. 65 (4), 283-290 (2012).

[7] Manev, H.: On the structure tensors of almost of contact B-metric Manifolds. Filomat. 29(3), 427-436 (2015).

[8] Morimoto, A.: On normal almost contact structures. J. Math. Soc. Japan. 15, 420-436 (1963).

[9] Özdemir, N., Solgun, M., Aktay, Ş.: Almost contact metric structures on 5-dimensional nilpotent Lie algebras. Symmetry. 76(8), (2016).

\section{Affiliations}

\section{ŞENAY BULUT}

ADDRESS: Eskişehir Technical University, Department of Mathematics, 26470, Eskişehir-Turkey. E-MAIL: skarapazar@eskisehir.edu.tr ORCID ID: 0000-0002-0317-1791

\section{SEVGI ENVEŞ ERMIŞ}

ADDRESs: Eskişehir Technical University, Department of Mathematics, 26470, Eskişehir-Turkey.

E-MAIL: sevgienvesermis@gmail.com

ORCID ID: 0000-0002-5523-3157 